\title{
Statistical Analysis of Native Contact Formation in the Folding of Designed Model Proteins
}

\author{
Guido Tiana and Ricardo A. Broglia \\ Department of Physics, University of Milano, \\ via Celoria 16, 20133 Milano, Italy, \\ INFN, Sez. di Milano, Milano, Italy, \\ The Niels Bohr Institute, University of Copenhagen, \\ Bledgamsvej 17, 2100 Copenhagen, Denmark
}

(October 26, 2018)

\begin{abstract}
The time evolution of the formation probability of native bonds has been studied for designed sequences which fold fast into the native conformation. From this analysis a clear hierarchy of bonds emerge a) local, fast forming highly stable native bonds built by some of the most strongly interacting amino acids of the protein, b) non-local bonds formed late in the folding process, in coincidence with the folding nucleus, and involving essentially the same strongly interacting amino acids already participating in the fast bonds, c) the rest of the native bonds whose behaviour is subordinated, to a large extent, to that of the local- and non-local native contacts.
\end{abstract}

\section{INTRODUCTION}

There is strong experimental evidence which testifies to the fact that the folding of small, single domain proteins can be pictured as the crossing of a free energy barrier between the unfolded state $\mathrm{U}$ (or, for some proteins like e.g. barnase [1], a populated intermediate state 
I which is fast at equilibrium with the unfolded state $\mathrm{U}$ ) and the native state $\mathrm{N}$ [2]. In any case, experiments usually detect one single time scale, which can be interpreted as the characteristic time $\tau \sim \exp (\Delta F / k T)$ needed by the system to cross the free energy barrier of height $\Delta F$ at a temperature $T, k$ being the Botzmann constant.

Under such circumstances it would seem rather uninteresting to develop models which describe the time evolution, in the folding process, of individual native contacts, if nothing else because whatever results one would obtain could be hardly checked experimentally, to the extent that the hierarchy of events which could eventually be found should express themself through a two-state picture. On the other hand, while this barrier crossing picture describes correctly a great deal of small proteins, it is not helpful in explaining how proteins can find their native state on very short times (tipically ranging from milliseconds to seconds) in spite of the enormous size of conformational space. This problem is usually referred to as Levinthal's paradox [3]. To address this problem is the main goal of the present work.

We have found that designed proteins follow a well defined hierarchy of steps en route to folding [4], which involve local elementary structures and the formation of a folding nucleus [5]. These steps answer Levinthal's paradox providing at the same time a microscopic picture to the well established two-state folding scenario. The final proof of the correctness of our results is to be found in the fact that they allow us to predict the 3D-structure of any designed model protein from its amino acid sequence [6], provided the contact energies used to design the sequence are known and an analytic expression which provide an overall account of the formation probability of the elementary structures involved in the folding process is available. The derivation of such an analytic expression is also one the goals of this work.

In Section 2 we review the model used to design the notional protein. In Section 3 we develop the analytic model of the time evolution of the native contacts and work out the solutions of the associated master equations. The value of the corresponding parameters are fixed by fitting these solutions with the numerical results of the simulations. In Section 4 we extract, from the results of the simulations, an analytic expression for the formation 
probability of the bonds. Chapter 5 contains the conclusions.

\section{THE MODEL}

A useful theoretical approach to study protein folding is a simplified lattice model, where the protein is a string of beads that is arranged on a cubic lattice [5, [7,8]. The configurational energy of a chain of $\mathrm{N}$ monomers is given by

$$
E=\frac{1}{2} \sum_{i, j}^{N} U_{m(i), m(j)} \Delta\left(\left|\vec{r}_{i}-\vec{r}_{j}\right|\right),
$$

where $U_{m(i), m(j)}$ is the effective interaction potential between monomers $m(i)$ and $m(j), \vec{r}_{i}$ and $\vec{r}_{j}$ denote their lattice positions and $\Delta(x)$ is the contact function. In Eq. (1) the pairwise interaction is different from zero when $i$ and $j$ occupy nearest-neighbour sites, i.e., $\Delta(a)=1$ and $\Delta(n a)=0$ for $n \geq 2$, where $a$ indicates the step length of the lattice. In addition to these interactions, it is assumed that on-site repulsive forces prevent two amino acids to occupy the same site simultaneously, so that $\Delta(0)=\infty$ (excluded volume

ansatz). The folding of the chain is simulated by Monte Carlo (MC) methods [9]. We shall consider throughout a 20-letters representation of protein sequence where $U$ is a $20 \times 20$ matrix. A possible realization of this matrix is given in ref. [10] (Table VI), where it was derived from frequencies of contacts between different amino acids in protein structures. The model we study here is a generic heteropolymer model which has been shown to reproduce important generic features of protein folding thermodynamics and kinetics, independent on the particular potential chosen [11,12]. This is achieved by using the same potential to design sequences and to simulate folding. However, in using such an approach, one should keep in mind that the labelling of amino acids (spherical beads all of the same size and with no side chain) is generic too and may be no obvious relation between those labels and labels for real amino acids.

Sequences are designed by minimizing, for fixed amino acid concentration, the energy of the native conformation with respect to the amino acid sequence. Good-folder sequences 
are characterized by a large gap $\delta=E_{c}-E_{n}$ (compared to the standard deviation $\sigma$ of the contact energies) between the energy of the sequence in the native conformation $E_{n}$, and the lowest energy of the conformations structurally dissimilar to the native conformation [13].

Examples of sequences designed to fold onto the conformation displayed in Fig. 1(a) are listed in Table 1. Among them, the sequences displaying $E_{n}=-16.5$ in the units we are considering $(0.6 \mathrm{kcal} / \mathrm{mol})$ [10 has been studied extensively in the literature [14 16] and is denoted $\mathrm{S}_{36}$. Very long, low temperature simulations indicated that $E_{c}=-14$. Consequently, for the designed sequence $\mathrm{S}_{36}, \delta=2.5 \gg \sigma=0.3$, a condition which guarantees fast folding [14. In fact, this sequence folds at $T=0.20$ in $8 \cdot 10^{8} \mathrm{MC}$ steps, while at $T=0.28$ it folds in the even shorter time of $6.5 \cdot 10^{5} \mathrm{MC}$ steps. The fractional population of the native state corresponding to these two temperatures is $91 \%$ and $10 \%$, respectively, to be compared with a population of 0.5 and of $10^{-5}$ for the heteropolymer folding temperatures of $T=0.25$ (temperature at which the probability for folding as well as for unfolding is $1 / 2$ ) and $T=0.40$ (temperature at which bonds break essentially as fast as they are formed due to thermal fluctuations) respectively. All the calculations discussed below, unless explicitely mentioned, were carried out at the temperature $T=0.28$, optimal from the point of view of allowing for the accumulation of representative samples of the different simulations, and at the same time leading to a consistent population of the native conformation.

We have discussed elsewhere [4] the results of folding simulations of designed sequences, which suggest the folding mechanism to be hierarchic. Let us here summarize the evolution of sequence $\mathrm{S}_{36}$. The starting point is an elongated, random generated conformation. After $\sim 10^{2}$ MCS two things have happened. The chain is now rather compact, having built of the order of 20 contacts (to be compared with the few contacts of the starting denaturated conformation and the maximum number of contacts for a full-compact 36mer, which is 40), as a consequence of the negative contact energies of the MJ interaction matrix (Table VI of ref. [10]). Also, three native contacts are already formed and rather stable (cf. Fig. 1(b)). They are the contacts between monomers 3-6, 11-14 and 27-30, which are both strongly interacting (their energy are $3.4 \mathrm{kT}, 2.7 \mathrm{kT}$ and $3.4 \mathrm{kT}$, respectively) and local (that is, close 
along the chain).

Afterwards, the system searches among rather compact conformations, until the native contacts among the local elementary structures (LES) formed by monomers 3-4-5-6, 27-2829-30 and 11-12-13-14 (which we call $\mathrm{S}_{4}^{1}, \mathrm{~S}_{4}^{2}$ and $\mathrm{S}_{4}^{3}$, respectively [17]) are built. This is the post-critical "folding nucleus" [5] and its formation takes $\sim 7 \cdot 10^{5} \mathrm{MCS}$. Once this is formed, the chain can reach the native conformation in $\sim 1000$ MCS. The key element determining the overall folding time, is the formation of the (post-critical) folding nucleus. However, this does not happen in an all-none fashion, but in a much more subtle and elaborated way, as testified by the dynamics of native bonds formation shown in Fig. 1(b). In fact, in the particular (and representative) case of $\mathrm{S}_{36}$, the folding nucleus is built in two steps, after the LES have been formed. First, the two LES $\mathrm{S}_{4}^{1}$ and $\mathrm{S}_{4}^{2}$ come together and occupy their native relative conformation in $\approx 10^{5} \mathrm{MCS}$. In the successive $4-5$ hundred thousands steps the partially formed folding nucleus is surrounded by non-native neighbours. When the remaining LES $\mathrm{S}_{4}^{3}$ finds its native position, the folding nucleus is completed and the chain folds almost immediately afterwards.

\section{STATISTICAL ANALYSIS OF NATIVE CONTACT FORMATION}

To provide a quantitative basis to discuss the behaviour observed in the snapshots of folding events discussed in the previous section, we have carried out a systematic study of native bonding statistics. For this purpose, the main quantity studied was the probability $P_{i, j}(t)$ that the $i$ th monomer forms its native contact with the $j$ th monomer as function of time, quantity found as average over many time evolutions. In every time evolution, the chain starts from a random conformation and the simulation is carried out over $10^{6} \mathrm{MC}$ steps.

From this analysis we have found that the 40 native bonds of sequence $\mathrm{S}_{36}$ can be classified in three groups according to the behaviour of the associated function $P_{i, j}(t)$ (see Fig. 2(b),

(c) and (d) ). The first group is composed of bonds which reach a stability considerably 
higher than all the other contacts $\left(P_{i-j} \approx 1\right)$ on short call. Bonds $3-6,11-14$ and $27-30$ (which we call "fast bonds") belong to this class and become stable in a time scale of the order of $10^{3}$ MCS. A second set of bonds (which we call "slow bonds") acquire a $0.5-0.8$ stability in a time scale of order of $10^{4}-10^{5} \mathrm{MC}$ steps. The majority of bonds reach a stability smaller than 0.5 in a time scale which is again of the order of $10^{5} \mathrm{MC}$ steps.

In order to understand the behaviour of $P_{i, j}$, we assume that each bond $i-j$ can be found in one of three possible states. It can be in its native state (which we will denote "N"), it can be unbound ("0") or its residues can be bound to some non-native residues ("W"). We also assume that the system can move between "0" and "N", between "0" and "W", but not between "N" and "W". The state "0" will denote not only the cases in which monomers $i$ and $j$ have no nearest neighbours at all, but also the cases in which their nearest neighbours interact weakly. This is the case when in a globule conformation a monomer moves to break a bond. If the density of monomers is high, in fact, it is unlikely that it can move into an isolated site. Nonetheless, this monomer will become neighbour of monomers which have no energetic preference for it (so they can be considered "random" monomers) and consequently the interaction energy between the moved monomer and its new neighbours is, in first approximation, zero.

According to this model it is possible to write, for each bond, the master equations

$$
\begin{aligned}
& \dot{P}_{n}(t)=w_{0 \rightarrow n} P_{0}(t)-w_{n \rightarrow 0} P_{n}(t), \\
& \dot{P}_{w}(t)=w_{0 \rightarrow w} P_{0}(t)-w_{w \rightarrow 0} P_{w}(t), \\
& P_{0}(t)=1-P_{n}(t)-P_{w}(t),
\end{aligned}
$$

where $P_{n}(t), P_{w}(t)$ and $P_{0}(t)$ are the probabilities that the bond under study is in its native state, in a non-native state or in a unbound state, respectively. The initial conditions for folding are that bonds start in an unbound state, so that $P_{0}(0)=1$.

To solve Eqs. (2), it is necessary to know how the transition probabilities $w_{0 \rightarrow n}, w_{n \rightarrow 0}$, $w_{0 \rightarrow w}$ and $w_{w \rightarrow 0}$ for a given bond depend on the states of all other bonds. We will show that the transition probabilities associated with "fast" bonds are essentially independent on 
the state of the other bonds, while the probabilities associated with "slow" bonds depend on the state of "fast" bonds. However, since "fast" bonds reach an equilibrium probability close to 1 in a time scale which is three orders of magnitude faster than that associated with "slow" bonds, it is possible to neglect this dependence. Although the situation is more complicated for the other bonds, as it will be clear later, the folding rate of the designed protein is essencially independent of them. In any case, one should notice that the transition probabilities $w_{0 \rightarrow n}, w_{n \rightarrow 0}, w_{0 \rightarrow w}$ and $w_{w \rightarrow 0}$ represent average values, and consequently do not depend on the actual conformation.

The probability that a native bond is formed is given by the solution of Eqs. (2), that is,

$$
P_{n}(t)=\alpha(1-\exp (-\lambda t))+\beta(1-\exp (-\mu t))
$$

where

$$
\begin{aligned}
& \lambda= \frac{1}{2}\left(w_{0 \rightarrow n}+w_{n \rightarrow 0}+w_{0 \rightarrow w}+w_{w \rightarrow 0}+\right. \\
&+\left(\left(w_{0 \rightarrow n}+w_{n \rightarrow 0}+w_{0 \rightarrow w}+w_{w \rightarrow 0}\right)^{2}-\right. \\
&\left.\left.+4\left(w_{0 \rightarrow n} w_{w \rightarrow 0}+w_{n \rightarrow 0} w_{w \rightarrow 0}+w_{n \rightarrow 0} w_{0 \rightarrow w}\right)\right)^{1 / 2}\right), \\
& \mu= \frac{1}{2}\left(w_{0 \rightarrow n}+w_{n \rightarrow 0}+w_{0 \rightarrow w}+w_{w \rightarrow 0}-\right. \\
&+\left(\left(w_{0 \rightarrow n}+w_{n \rightarrow 0}+w_{0 \rightarrow w}+w_{w \rightarrow 0}\right)^{2}-\right. \\
&\left.\left.+4\left(w_{0 \rightarrow n} w_{w \rightarrow 0}+w_{n \rightarrow 0} w_{w \rightarrow 0}+w_{n \rightarrow 0} w_{0 \rightarrow w}\right)\right)^{1 / 2}\right), \\
& \alpha=\frac{1}{\lambda-\mu}\left(\frac{-\mu w_{0 \rightarrow n} w_{w \rightarrow 0}}{w_{0 \rightarrow n} w_{w \rightarrow 0}+w_{n \rightarrow 0} w_{w \rightarrow 0}+w_{n \rightarrow 0} w_{0 \rightarrow w}}+w_{0 \rightarrow n}\right), \\
& \beta=\frac{1}{\lambda-\mu}\left(\frac{\lambda w_{0 \rightarrow n} w_{w \rightarrow 0}}{w_{0 \rightarrow n} w_{w \rightarrow 0}+w_{n \rightarrow 0} w_{w \rightarrow 0}+w_{n \rightarrow 0} w_{0 \rightarrow w}}-w_{0 \rightarrow n}\right) .
\end{aligned}
$$

The set of parameters $\lambda, \mu, \alpha$ and $\beta$ associated with each of the native bonds of sequence $\mathrm{S}_{36}$ were determined through a least-square fit of the results of the MC simulations (cf. e.g. Figs. 2(b), 2(c) and 2(d) ). From these values of $\lambda, \mu, \alpha$ and $\beta$ the transition probabilities $w_{0 \rightarrow n}, w_{n \rightarrow 0}, w_{0 \rightarrow w}$ and $w_{w \rightarrow 0}$ were determined with the help of Eqs. (4-7). 


\section{A. Fast Bonds}

The bonds between monomers $3-6,11-14$ and $27-30$ are formed remarkably fast. The parameters of Eq. (2), fitted in the range $0-1000$ of MC steps, and the transition rates derived from them are shown in Table 2. The standard deviation between the fitting function and the data is $9.2 \cdot 10^{-5}$, indicating that Eq. (2) describes accurately the bonding of these monomers. According to this relation the bonding process takes place with two different characteristic times, namely $1 / \lambda$ and $1 / \mu$, depending on whether the monomers involved can form the bond directly, or get trapped in non-native bonds ("w"). The order of magnitude of the overall process can be summarized by the characteristic time $\tau=(\alpha \lambda)^{-1}+(\beta \mu)^{-1}$. The characteristic times $\tau$ for fast bonds under discussion are of the order of $10^{2}$ MCS (Table 3), a very short time as compared to the average first passage folding time of $7 \cdot 10^{5} \mathrm{MC}$ steps.

The effective bonding free energy $\Delta F$ can be derived from the expression $w_{n \rightarrow 0}=$ $w_{0} \exp (\Delta F / T), w_{0}$ being the inverse of the time step. Since at each MC step before folding, every monomer is attempted to move with equal probability [18], it is reasonable to chose $w_{0}=2 / N, N$ being the length of the chain and the factor 2 keeps into account that it is possible to create or break a bond moving one of its two monomers. The values of $\Delta F$ for the three "fast" bonds are $\Delta F=-1.05, \Delta F=-1.01$ and $\Delta F=-1.15$, respectively. The bonding free energy is $\Delta F=F_{n}-F_{0}=E_{n}-E_{0}-T S_{n}+T S_{0}$, where $E_{n}, E_{0}(=0), S_{n}$ $(=0)$ and $S_{0}$ are the contact energies and entropies associated with the native and with the unbound states. In the case of bond 3-6, where $E_{n}-E_{0}=-0.97$ (similar values are associated with the other fast bonds), one obtains $S_{0} \approx 0$. Consequently, there is in average only one non-bound state available to the system, due to the excluded volume constrain (to be noted that in the present simulations, the chain starts from a random generated conformation, which is usually rather compact). If the chain was in a non-compact conformation, there would be 9 possible unbound states for bonds between monomers $i$ and $i+3$, which would lead to an entropy equal to $S_{0}=2.19$ and, consequently, to an overall stability of 
$\alpha+\beta=0.35$ (instead of 0.77 ). According to the present model, it is necessary a collapse of the chain to a compact conformation before the fastest native bonds are stabilized [19]

On a time scale of $10^{5} \mathrm{MC}$ steps, fast bonds $3-6,11-14$ and 27-30 reach a stability of $\alpha+\beta=0.97, \alpha+\beta=0.91$ and $\alpha+\beta=0.96$, respectively (instead of $0.77,0.76$ and 0.65 , as measured on a $10^{3}$ timescale). This increment in stability can only come from the interaction with other monomers which is, as we shall see, the case.

\section{B. Slow Bonds}

The distributions $P_{n}(t)$ related to the bonds acting among the set of monomers $(3,4,5,6)$, $(11,12,13,14)$ and $(27,28,29,30)$ (which we have called "slow" bonds) display a remarkable similarity. The parameters associated with some of these distributions are listed in Table 4 . Again, the functions $P_{n}$ are well described by Eq. (2), the average standard deviation from the fitted function being $2.2 \cdot 10^{-5}$. The dependence of the transition rates associated with these bonds on the state of "fast" bonds is negligible, since "fast" bonds reach equilibrium (with $P_{w}(\infty) \sim 1$ ) in a time which is 3 orders of magnitude shorter than the relaxation time of "slow" bonds. The characteristic bonding times $\tau$ for these bonds range from $4.4 \cdot 10^{4}$ to $2.8 \cdot 10^{5}$ MC steps.

The unbinding free energy for the "slow" bonds, calculated in the same way as for the "fast" bonds, ranges from -1.57 to -2.43 . The bond $28-5$, for example, has $\Delta F=-2.61$ and the monomers interact with a potential energy $E=-0.41$. Assuming again that the native state is unique, this bond should satisfy

$$
-0.41+T S_{0}=-2.61
$$

which is impossible, because it gives $S_{0}=-7.3$ (and $S_{w}=-1.93$, setting $E_{w} \approx-\sigma=-0.3$ )

while the entropy of the unbound state $S_{0}$ must be a positive quantity. On the other hand, we can consider that the monomers $(3,4,5,6)$ form a rigid structure (the bond $3-6$ is very stable) that interact, as a whole, with the structure built of the monomers $(27,28,29,30)$ 
(the bond $27-30$ is also very stable). In this case, the total energy involved is the sum of the four energies of the bonds associated with the 8 monomers contained in the two structures, that is $E=-3.02$. Thus, Eq. (8) becomes

$$
-3.02+T S_{0}=-2.61,
$$

which is satisfied if $S_{0}=1.46$, leading to $\approx 4$ unbound states. The same argument applies when considering the interaction, as a whole, between the monomers $(11,12,13,14)$ and those belonging to the other two structures.

From the above results, one identify the "fast" bonds with those which stabilize the local elementary structures discussed in Section 2 and "slow" bonds as those among local elementary structures.

\section{The other bonds}

So far, only very favorably interacting bonds have been studied, whose interaction energy is between -0.7 and -1 . The majority of bonds, however, have higher energy, sometimes even positive. In Fig. 2(d) it is displayed the behaviour of $P(t)$ associated with the bond $24-15$, whose interaction energy is -0.38 , as a function of time. The fit with the function given in Eq. (2) leads to $w_{n \rightarrow 0}=1.3 \cdot 10^{-5}$. It follows that the free energy which keeps the two residues bound is $\Delta F=-2.14$, quantity which can be decomposed as $\Delta F=E_{n}+T S_{0}$, in keeping with the fact that $E_{0}=S_{n}=0$. This cannot hold for any positive value of $S_{0}$, implying that there is an effective force, due to the correlation with other residues, which decreases the value of $E_{n}$ below -0.38 .

Since this class of weakly interacting bonds, unlike "slow bonds", are not associated with stable substructures, correlations among them can only be due to the polymeric constrain, after "fast" and "slow" bonds have reached the native state, building a stable folding nucleus. Under such circumstances the remaining monomers form short loops which begin and end in

the folding nucleus. The space available to these residues is small and the polymeric bonds 
keep them together in such a way that each loop interact cooperatively with the rest of the chain (even if they are less rigid than the local elementary structures). In the case of bond 24-15, for example, monomer 15 is almost fixed once the core has been stabilized, being constrained, to a large extent, by residue 14 belonging to the core. Monomer 24 belongs to a loop which extends from monomer 16 to monomer 27 , and is thus affected by the interactions of all these monomers among themselves and with the core.

If this scenario is correct, then the fit of $P(t)$ obtained making use of Eqs. $(3-7)$ is not correct, since the parameters of the master equations now depend on time, leading to a more complicated solution. Assuming for example that the rate $w_{0 \rightarrow n}$ has a time dependence in the form $w_{0 \rightarrow n}(t)=w_{0 \rightarrow n}^{\prime}(1-\exp (-\nu t))$, with $\nu=4 \cdot 10^{-5}$, the typical formation time of slow bonds, we found $\Delta F=-1.58$. The characteristic bonding time for the bond $24-15$ is $\tau=2.6 \cdot 10^{5}$ (other times are, i.e., $\tau=3.3 \cdot 10^{5}$ for bond $7-10$ and $\tau=3.2 \cdot 10^{5}$ for bond 10-15). This is very fast, considering that in that time it is included the bonding time of "slow" bonds, on which it depends. At the basis of this result one finds the decrease in entropy caused by the formation of the folding nucleus, an event which not only contribute to stabilizing these weak bonds, but also make their formation faster.

Summing up, the chain first builds strongly interacting local contacts $(3-6,11-14$ and 27-30) in a time which is of the order of 100 MCS. The structures built in this way ("local elementary structures") behave as elementary entities, which are strongly interacting, have a small conformational space available and are unlikely to form stable non-native bonds. These local elementary structures come to their native position in a time of the order of $10^{5}$ MCS, building the folding nucleus of the protein. Almost immediately hereafter, the weak residues fold to their native position, due to the reduction of the conformational space available to them and to the strong effective force that the polymeric chain produces. 


\section{Hindsight}

Folding seems to take place as a hierarchical succession of events. Why this succession of events makes folding fast? Let us consider the number of conformations available at each level of the folding process (cf. Fig. 3). At the beginning there are no constrains to the system and the chain can assume $10^{24}$ different conformations. After the chain has collapsed to a compact globule, there are $10^{19}$ conformations. The formation of the LES, which is a local event, and consequently does not require a massive search in conformation space, further reduces the number of conformations to $10^{14}$. The native bonding of LES $\mathrm{S}_{4}^{1}$ and $\mathrm{S}_{4}^{2}$ further decrease the size of conformational space to $10^{11}$, while only $10^{5}$ conformations are associated to the folding nucleus. Consequently, at each step of the hierarchy of events bringing the heteropolymer from the denaturated state to the native state, the system has to search only among a number of conformations given by the ratio between the number of conformations associated with the step in question and the successive step. In this way entropy is slowly squeezed out of the chain, until the system reaches the unique ground state, with zero entropy. One can notice that the most important step in terms of the decrease in entropy of the system is the step associated with the completion of the folding nucleus, i.e. the step in which the LES $\mathrm{S}_{4}^{3}$ forms its native contacts with the partially formed nucleus made out of the LES $\mathrm{S}_{4}^{1}$ and $\mathrm{S}_{4}^{2}$, during which the system has to search among $10^{6}$ conformations. To be noted that to this step is associated a rather small gain in energy (2.13, to be compared with 3.02 associated with the binding of local elementary structures $\mathrm{S}_{4}^{1}$ and $\mathrm{S}_{4}^{2}$ ). This is likely the top of the free energy barrier separating the native from the unfolded states.

The importance in the folding process of a designed sequence of local elementary structures is multiple, in that they decrease the entropy of the chain, enhance the elementary contact energies (from pairs of monomers to pairs of LES) and reduce the number of possibilities in which the most strongly interacting residues can assemble together. The decrease in entropy is due to the fact that elementary structures are rather stable, so that all the 
residues belonging to an elementary substructure behave effectively as a single residue and the chain becomes, effectively, shorter. Moreover, while contact energies among the single, strongest interacting, amino acids are of the order of $-1=-3.6 k T$ [10], the interaction between LES built out of four of these amino acids (e.g. $S_{4}^{1}$ and $S_{4}^{2}$ ) is four times stronger $(\approx-4=-13 k T)$. Finally, wrong (non-native) contacts are more probable to be established during the folding process between single strongly interacting monomers, than between local elementary structures. The set of local elementary structures is like a 4-digits code. To create a strong bond, all the four monomers have to match. After the local elementary structures have come to occupy their native position in the protein, the chain is composed by a nucleus surrounded by short loops. Each loop can build its native bonds in a very short time due to its small entropy, leading the chain to the native state.

For other sequences, the same mechanism has been found to be at the basis of the folding process, even if the LES can now assume different shapes. For example, folding of sequence number 8 of Table 1, to the conformation shown in Fig. 1(a), is controlled by the local elementary structures formed by monomers 1-2, 16-21 and 31-32. In this case, a LES is larger than those associated with $\mathrm{S}_{36}$, involving 6 monomers, and two of them are "degenerate", being composed of just two monomers, the peptide bond playing the role of the strong interaction which gives life to the elementary substructure. The case of the sequence folding to the conformation displayed in Fig. 4(a) (cf. caption to the figure) is rather peculiar, in the sense that this native conformation has been design to minimize the amount of local contacts [20]. Also in this case folding is lead by local elementary structures, which are 1-6, 30-31 and 20-22 (cf. Fig. 4(b) ). A further example, associated with a chain made of 48 monomers, is displayed in Fig. 4(c). To the sequence folding to this conformation and listed in the caption, correspond rather large local elementary structures, built of monomers 2-12 and 33-41 (in Fig. 4(d) it is displayed the bond dynamics for this sequence). 


\section{DISCUSSION}

The results collected in Tables 2 and 4 testify to the fact that the two main features which characterize the contacts as belonging to the three different groups of native bonds are their locality (namely, their distance along the chain) and their interaction energies. "Fast bonds" are local and strongly interacting, "slow bonds" are non-local and strongly interacting, while the other bonds are weakly interacting.

Whether a bond belongs to a group or to another is determined by the values of the jumping rates $w_{0 \rightarrow n}, w_{n \rightarrow 0}, w_{0 \rightarrow w}$ and $w_{w \rightarrow 0}$. The rate $w_{0 \rightarrow n}$ can be thought as having a purely geometrical dependence. In Fig. 5 we display the inverse of $w_{0 \rightarrow n}$ associated with the sequence $\mathrm{S}_{36}$ as a function of the distance $|j-i|$, measured along the chain, between the two monomers involved in the bond. The monotonic behaviour of the values of $w_{0 \rightarrow n}$ is compared with the inverse probability that the $i$ th and $j$ th monomers come close during a random search (dotted curve in Fig. 5). Such probability is simply the number of conformations $\gamma^{N-2}(j-i)^{-1.68}, \gamma$ being the effective coordination number of the cubic lattice [21], in which the $i$ th monomer is nearest neigbours of the $j$ th monomer divided by the total number of conformations $\gamma^{N-1}$ of the chain. The above expression for the number of conformations with a bond between the $i$ th and $j$ th monomer is given by the product of three terms associated with the corresponding parts which compose each conformation, namely two branches of lenght $i$ and $N-j+1$ respectively, and a loop of length $j-i$. The first two terms contribute a factor $\gamma^{i-1}$ and $\gamma^{N-j}$ respectively. The contribution $\gamma^{j-i-1}(j-i)^{-1.68}$ associated with the loop was determined through a fit of the number of conformations obtained by a complete enumeration up to 23] $j-i=16$ 24].

While it is not easy to derive $w_{0 \rightarrow w}$ from theoretical considerations, it is correlated to $w_{0 \rightarrow n}$ (at least, as order of magnitude (see Tables 2 and 4$)$ ). On the other hand, $w_{w \rightarrow 0}$ is approximately constant $\left(1.9 \cdot 10^{-2}\right.$ for fast bonds and $4.6 \cdot 10^{-5}$ for the other bonds).

The parameter $w_{n \rightarrow 0}$ depend on the free energy difference between the native bond and the unbound state. For "fast" bonds the change in internal energy is given by the associated 
matrix elements, depending only on the kind of the pairs of monomers involved. Apparently, the change in entropy between unbound and native states is negligible for these bonds. In the case of "slow" bonds, that is bonds among local elementary structures, one has to consider the change in internal energy of all the monomers belonging to the structures, while the entropy is rather constant $\left(S_{0}=-2.43 \pm 0.09\right)$.

\section{CONCLUSIONS}

The time evolution of the probability of native bond formation has been studied for designed sequences which fold fast into the native conformation. From this analysis we have found that the native bonds can be classified in three groups namely, ("fast") bonds which reach a stability considerably higher than all the other contacts (and close to 1, at "biological" temperatures) in a time scale of the order of $10^{3} \mathrm{MC}$ steps, ("slow") bonds which acquire $0.5-0.8$ stability in a time scale of the order of $10^{4}-10^{5} \mathrm{MC}$ steps, and bonds (the majority) which reach a stability smaller than 0.5 in a time scale which is again

of the order of $10^{5} \mathrm{MC}$ steps. The first two types of bonds correspond to the (few) local and the (relatively many) non-local contacts found in the (post-critical) folding nucleus. Fast and slow contacts completely control the folding process. These results provide a simple picture of the folding process, where local elementary structures, stabilized by the strong, fast native bonds build, by assembling together, the slow bonds (folding nucleus), after which the remaining bonds fall in place within a very short time.

\section{Acknowledgments}

We are grateful to Anke Odermann for the data concerning the full enumeration used in Chapt. 4. 


\section{REFERENCES}

[1] A. R. Fersht, Phil. Trans. R. Soc. Lond. B 348, 11 (1995)

[2] A. R. Fersht, Structure and Mechanism in Protein Science, W. H. Freeman and Co., New York (1999)

[3] C. Levinthal, J. Chem. Phys. 65, 44 (1968)

[4] R. A. Broglia and G. Tiana, cond-mat/0003096 (to be published)

[5] V. I. Abkevich, A. M. Gutin and E. I. Shakhnovich, Biochemistry 33, 10026 (1994)

[6] R. A. Broglia and G. Tiana, cond-mat/0005150 (to be published)

[7] N. Go, Int. J. Peptide Prot. Res. 7, 313 (1975)

[8] K. F. Lau and K. Dill, Macromolecules 22, 3986 (1989)

[9] J. Rey and J. Sklonik, Chem. Phys. 158, 199 (1991)

[10] S. Miyazawa and R. Jernigan, Macromolecules 18, 534 (1985)

[11] E. I. Shakhnovich, Curr. Opin. Struct. Biol. 7, 29 (1997)

[12] E. I. Shakhnovich, Folding and Design 1, R50 (1996)

[13] A. Sali, E. I. Shakhnovich and M. Karplus, J. Mol. Biol. 235, 1614 (1994)

[14] V. I. Abkevich, A. M. Gutin and E. I. Shakhnovich, J. Chem. Phys 101, 6052 (1994)

[15] D. K. Klimov and D. Thirumalai, J. Mol. Biol 282, 471 (1998)

[16] G. Tiana, R. A. Broglia, H. E. Roman, E. Vigezzi and E. I. Shakhnovich, J. Chem. Phys. 108, 757 (1998)

[17] R. A. Broglia, G. Tiana, S. Pasquali, H. E. Roman and E. Vigezzi, Proc. Natl. Acad. Sci. USA 95, 12930 (1988)

[18] One has to remember that move attempts have not to violate the double occupancy 
restriction, so it is not trivial that all monomers are attempted to move with the same probability. In particular, after the chain has reached its native state, this is no longer true, in that the movement of bulk sites is attempted with a lower probability rather than surface sites.

[19] This seems not to be true if the interaction matrix is changed to a new one whose elements are, in average, repulsive (cf. ref [5]).

[20] V. I. Abkevich, A. M. Gutin and E. I. Shakhnovich, J. Mol. Biol. 252, 460 (1998)

[21] P. J. Flory, J. Chem. Phys 17, 303 (1949)

[22] P.-J. de Gennes, Scaling concepte in polymer physics, Cornell Univeristy Press, New York, 1979

[23] For longer loops one can use the standard analytical expressions available in the literature (cf. e.g. ref. [22])

[24] A. Odermann, (private communication) 


\section{TABLES}

\begin{tabular}{|l|l|l|}
\hline \hline 1 & -17.13 & YPDLTKWHAMEAGKIRFSVPDACLNGEGIRQVTLSN \\
2 & -16.50 & SQKWLERGATRIADGDLPVNGTYFSCKIMENVHPLA \\
3 & -16.38 & GNRLPESGAAKGHELDFAWGTLVLSQKYIDNMRVPS \\
4 & -16.32 & TGKVQEGAIERSNDLDMAAGTLCIHPKPLESWRVYN \\
5 & -15.27 & NQEPLKRNGRDCARGTLYVSHGPFDVEIMKTIAWLA \\
6 & -15.24 & PPKVLERQTGNAINGDFDAYRGSCWLKLVEHTSAIM \\
7 & -14.91 & PENLFERQVWHTGDMDIPCYRVGLSAKGINKLTASA \\
8 & -14.06 & DKYAIPDRTTNLVNGEFHVKRGCGSMQLSPELWAIA \\
\hline
\end{tabular}

TABLE I. Eigth sequences obtained through a Metropolis Monte Carlo optimization in the space of sequences at different "selective" temperatures. The conformation onto which they have been minimized is the one displayed in Fig. 1(a). The second sequence of the list is known in the literature [14-16] as $\mathrm{S}_{36}$.

\begin{tabular}{|c|r|r|r|r|}
\hline \hline bond & $\alpha$ & $\lambda$ & $\beta$ & $\mu$ \\
\hline $3-6$ & 0.19 & 0.15 & 0.58 & 0.0047 \\
$27-30$ & 0.25 & 0.07 & 0.51 & 0.0049 \\
$11-14$ & 0.38 & 0.023 & 0.27 & 0.0017 \\
\hline \hline
\end{tabular}

\begin{tabular}{|c|c|c|c|c|}
\hline \hline \hline bond & $w_{0 \rightarrow n}$ & $w_{n \rightarrow 0}$ & $w_{0 \rightarrow w}$ & $w_{w \rightarrow 0}$ \\
\hline $3-6$ & $3.1 \cdot 10^{-2}$ & $1.3 \cdot 10^{-3}$ & $1.0 \cdot 10^{-1}$ & $1.7 \cdot 10^{-2}$ \\
$27-30$ & $1.9 \cdot 10^{-2}$ & $1.5 \cdot 10^{-3}$ & $4.0 \cdot 10^{-1}$ & $1.3 \cdot 10^{-2}$ \\
$11-14$ & $9.1 \cdot 10^{-3}$ & $9.3 \cdot 10^{-4}$ & $1.1 \cdot 10^{-2}$ & $2.7 \cdot 10^{-3}$ \\
\hline \hline
\end{tabular}

TABLE II. The fitted parameters and the related jumping rates for "fast" bonds done on the time range $0-1000 \mathrm{MC}$ steps, derived from these parameters making use of Eqs. 4-7. The standard deviation of the fitted curve from the data is $9.2 \cdot 10^{-5}$. 


\begin{tabular}{|c|r|r|r|}
\hline \hline bond & $\tau$ & bond & $\tau$ \\
\hline $3-6$ & 401 & $14-27$ & $1.7 \cdot 10^{5}$ \\
$27-30$ & 457 & $6-11$ & $2.5 \cdot 10^{5}$ \\
$11-14$ & 2292 & $28-13$ & $1.9 \cdot 10^{5}$ \\
$28-5$ & $1.3 \cdot 10^{5}$ & $15-24$ & $2.6 \cdot 10^{5}$ \\
$27-6$ & $1.2 \cdot 10^{5}$ & $7-10$ & $3.3 \cdot 10^{5}$ \\
$4-29$ & $4.4 \cdot 10^{4}$ & $10-15$ & $3.3 \cdot 10^{5}$ \\
$5-12$ & $2.0 \cdot 10^{5}$ & & \\
\hline \hline
\end{tabular}

TABLE III. Characteristic times for some selected bonds.

\begin{tabular}{|l|r|r|r|r|}
\hline \hline bond & $\alpha$ & $\lambda$ & $\beta$ & $\mu$ \\
\hline $28-5$ & 0.17 & $1.3 \cdot 10^{-4}$ & 0.61 & $1.9 \cdot 10^{-5}$ \\
$27-6$ & 0.21 & $1.4 \cdot 10^{-4}$ & 0.60 & $2.0 \cdot 10^{-5}$ \\
$4-29$ & 0.44 & $7.3 \cdot 10^{-5}$ & 0.39 & $1.9 \cdot 10^{-4}$ \\
$5-12$ & 0.26 & $9.7 \cdot 10^{-4}$ & 0.24 & $2.1 \cdot 10^{-5}$ \\
$14-27$ & 0.25 & $1.4 \cdot 10^{-4}$ & 0.40 & $1.7 \cdot 10^{-5}$ \\
$6-11$ & 0.46 & $7.8 \cdot 10^{-4}$ & 0.21 & $1.9 \cdot 10^{-5}$ \\
$28-13$ & 0.11 & $3.7 \cdot 10^{-4}$ & 0.34 & $1.7 \cdot 10^{-5}$ \\
\hline \hline
\end{tabular}




\begin{tabular}{|l|c|c|c|c|}
\hline \hline bond & $w_{0 \rightarrow n}$ & $w_{n \rightarrow 0}$ & $w_{0 \rightarrow w}$ & $w_{w \rightarrow 0}$ \\
\hline $28-5$ & $3.3 \cdot 10^{-5}$ & $4.9 \cdot 10^{-6}$ & $5.3 \cdot 10^{-5}$ & $5.7 \cdot 10^{-5}$ \\
$27-6$ & $4.1 \cdot 10^{-5}$ & $4.6 \cdot 10^{-6}$ & $5.9 \cdot 10^{-5}$ & $5.4 \cdot 10^{-5}$ \\
$4-29$ & $3.1 \cdot 10^{-5}$ & $1.0 \cdot 10^{-5}$ & $1.0 \cdot 10^{-5}$ & $1.6 \cdot 10^{-5}$ \\
$5-12$ & $2.5 \cdot 10^{-4}$ & $1.4 \cdot 10^{-5}$ & $6.8 \cdot 10^{-4}$ & $3.9 \cdot 10^{-5}$ \\
$14-27$ & $4.8 \cdot 10^{-5}$ & $7.7 \cdot 10^{-6}$ & $7.0 \cdot 10^{-5}$ & $3.7 \cdot 10^{-5}$ \\
$6-11$ & $3.6 \cdot 10^{-4}$ & $1.1 \cdot 10^{-5}$ & $3.9 \cdot 10^{-4}$ & $2.7 \cdot 10^{-5}$ \\
$28-13$ & $4.6 \cdot 10^{-5}$ & $1.0 \cdot 10^{-5}$ & $2.6 \cdot 10^{-4}$ & $6.0 \cdot 10^{-5}$ \\
\hline \hline
\end{tabular}

TABLE IV. The parameters related to the "slow" bonds, fitted in the range $0-10^{6}$ MC steps. The typical standard deviation for these fits is $2.2 \cdot 10^{-5}$. 


\section{FIGURES}

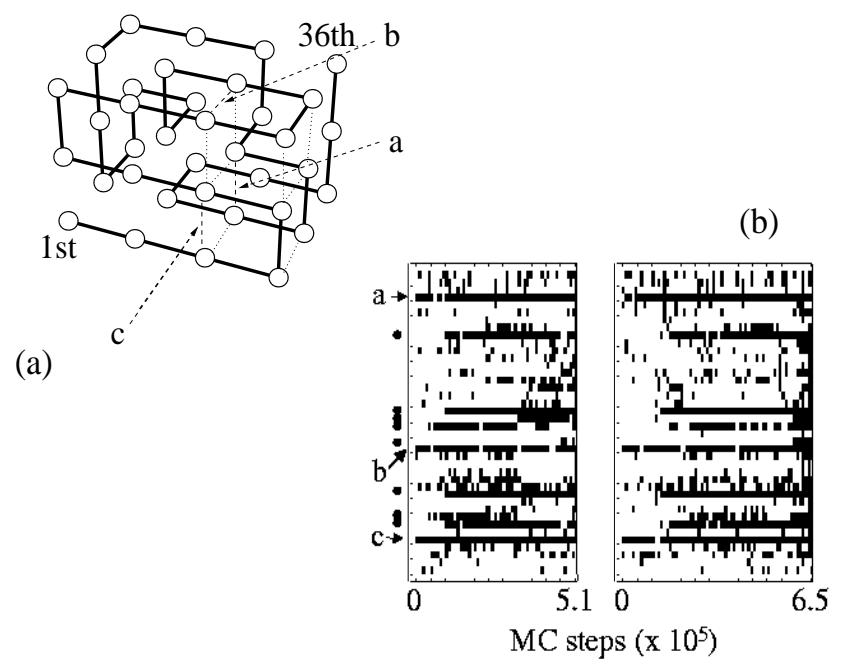

FIG. 1. (a) The native conformation of sequence SQKWLERGATRIADGDLPVNGTYFSCKIMENVHPLA (upper left). Bonds within local elementary structures (LES) are indicated with a dashed line, while bonds among local substructures with a dotted line. Examples of bonds among LES involve monomers 27-30, 11-14 and 3-6, and are marked a, b and c, respectively.(b) The dynamics of native contacts for two typical runs is shown in the lower right panels. On the x-axis is time, while to the y-axis are associated the 40 native contacts that this sequence can build. A black square in the plot indicates that a given bond is built at that time. Bonds stabilizing local elementary structures are marked with a, b and c, while bonds among local elementary structures are marked with solid dots.

(a)
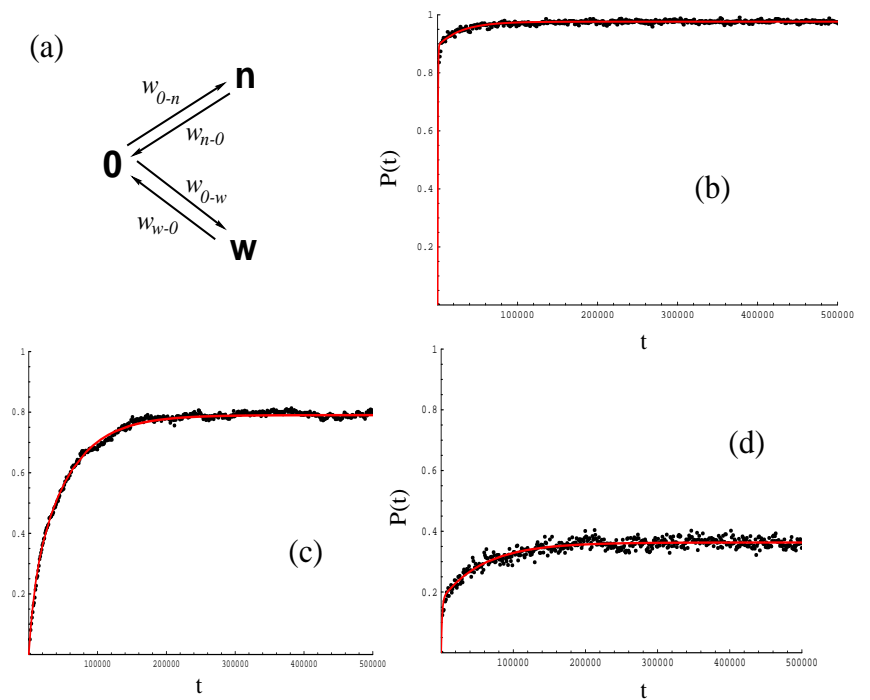
FIG. 2. (a) Each native contact can be though to be in its native state ("n"), in an unbound state ("0") or bound to some non-native monomer ("w"). This model is described by Eq. (1). (b) The probability $P_{i-j}(t)$ for the bond 3-6. To the curve obtained by simulations is superimposed the least-squares fit done with Eq. 2. (c) the same for bond 5-28. (d) The same for bond 15-24.

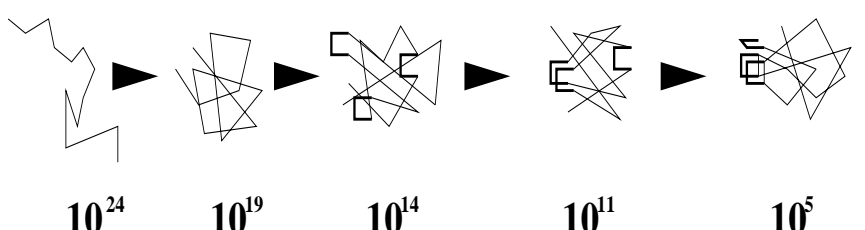

FIG. 3. Schematic representation of the mechanism according to which the system sqeezes out its entropy, by reducing the number of conformations available to it (indicated in lower part of the figure). The pieces of the heteropolymer drawn with heavy lines indicate the LES of the model protein.

(a)

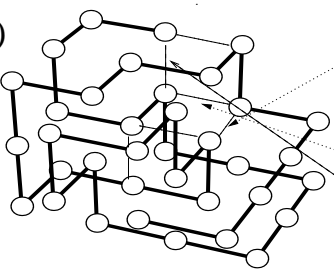

(c)

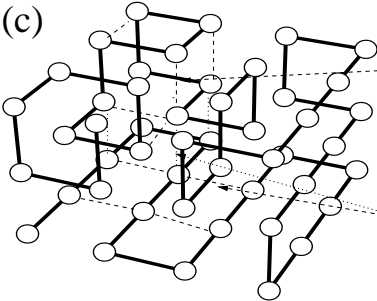

(b)

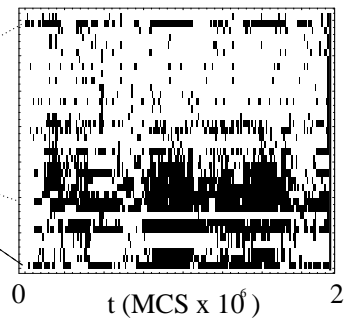

(d)

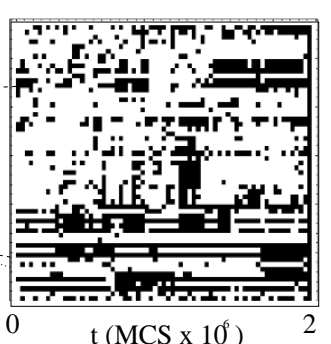

FIG. 4. The native states of the sequence RASMKDKTVGIGHQLYLNFEGEWCPAPDNTRVSLAI (a) and IMESQKWLCMEPAHWCVYTIQGLGNVNCPNTREFDSGRSKIQDAYLFH (c). The dynamics of the native bonds for these sequences are displayed in (b) and (d), respectively. 


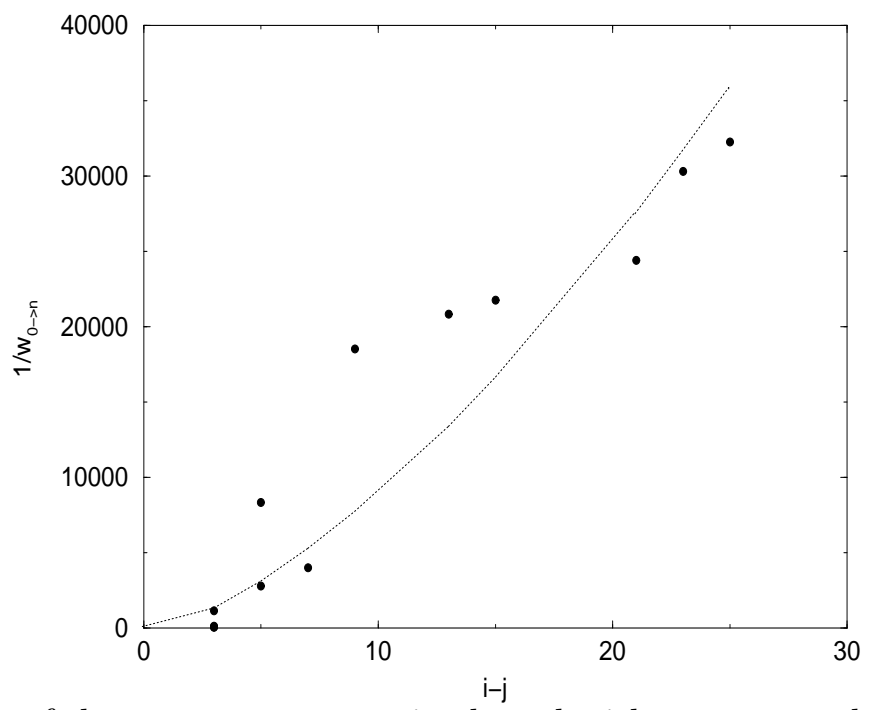

FIG. 5. The inverse of the parameter $w_{0 \rightarrow n}$ is plotted with respect to the distance $|i-j|$ of the monomers involved in the bond, measured along the chain (circles), compared to the probability that the two monomers come close during a random search (dotted line), normalized accordingly. 